\title{
LA:UR- $97-2644$
}

- Approved for public release; distribution is unlimited.

Title:

$C 0 N F-970967-$

Author(\$):

HANS E. HARTSE, GEOPHYSICS GROUP EES-3, MS C335, LOS ALAMOS NATIONAL LABORATORY

STEVEN R. TAYLOR, GEOPHYSICS GROUP EES-3, MS C335, LOS ALAMOS NATIONAL LABORATORY

WILLIAM S. PHILLIPS, GEOPHYSICS GROUP EES-3, MS C335, LOS ALAMOS NATIONAL LABORATORY

GEORGE E. RANDALL, GEOPHYSICS GROUP EES-3, MS C335, LOS ALAMOS NATIONAL LABORATORY

Submitted to:

PROCEEDINGS OF THE 19TH SEISMIC RESEARCH SYMPOSIUM ON MONITORING A COMPREHENSIVE TEST BAN TREATY.

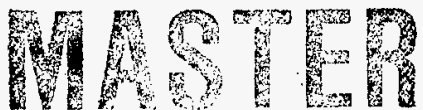

\section{DISTARSTHON OF THS DOCUMERT KS UNAMTED}

\section{Los Alamos}

NATIONALLABORATORY

Los Alamos National Laboratory, an affirmative action/equal opportunity employer, is operated by the University of California for the U.S. Department of Energy under Contract W-7405-ENG-36. By acceptance of this article, the publisher recognizes that the U.S. Government retains a nonexclusive, royalty-free license to publish or reproduce the published form of this contribution, or to allow others to do so, for U.S. Government purposes. Los Alamos National Laboratory requests that the publisher identify this article as work performed under the auspices of the U.S. Department of Energy. The Los Alamos National Laboratory strongly silpports academic freedom and a researcher's right to publish; as an institution, however, the Laboratory does not endorse the vieu point 


\section{DISCLAIMER}

This report was prepared as an account of work sponsored by an agency of the United States Government. Neither the United States Government nor any agency thereof, nor any of their employees, makes any warranty, express or implied, or assumes any legal liability or responsibility for the accuracy, completeness, or usefulness of any information, apparatus, product, or process disclosed, or represents that its use would not infringe privately owned rights. Reference herein to any specific commercial product, process, or service by trade name, trademark, manufacturer, or otherwise does not necessarily constitute or imply its endorsement, recommendation, or favoring by the United States Government or any agency thereof. The views and opinions of authors expressed herein do not necessarily state or reflect those of the United States Government or any agency thereof. 


\title{
APPLYING SOURCE AND PATH CORRECTIONS TO IMPROVE DISCRIMINATION IN CHINA
}

\author{
Hans E. Hartse, Steven R. Taylor, W. Scott Phillips, and George E. Randall \\ Geophysics Group - EES-3, Los Alamos National Lab
}

Sponsored by U.S. Department of Energy, Contract W-7405-ENG-36

Comprehensive Test Ban Treaty Research and Development Program, ST482A

\begin{abstract}
Monitoring the Comprehensive Test Ban Treaty (CTBT) to magnitude levels below 4.0 will require use of regional seismic data recorded at distances of less than $2000 \mathrm{~km}$. To improve regional discriminant performance we tested three different methods of correcting for path effects, and the third method includes a correction for source-scaling. We used regional recordings of broadband from stations in and near China. Our first method removes trends between phase ratios and physical parameters associated with each eventstation path. This approach requires knowledge of the physical parameters along an event-station path, such as topography, basin thickness, and crustal thickness. Our second approach is somewhat more empirical. We examine spatial distributions of phase amplitudes after subtracting event magnitude and correcting for path distance. For a given station, phase, and frequency band, we grid and then smooth the magnitude-corrected and distance-corrected amplitudes to create a map representing a correction surface. We reference these maps to correct phase amplitudes prior to forming discrimination ratios. Our third approach is the most complicated, but also the most rigorous. For a given station and phase, we invert the spectra of a number of well-recorded earthquakes for source and path parameters. We then use the values obtained from the inversion to correct phase amplitudes for the effects of source size, distance, and attenuation. Finally, the amplitude residuals are gridded and smoothed to create a correction surface representing secondary path effects.

We find that simple ratio-parameter corrections can improve discrimination performance along some paths (such as Kazakh Test Site (KTS) to WMQ), but for other paths (such as Lop Nor to AAK) the corrections are not beneficial. Our second method, the empirical path correction surfaces, improves discrimination performance for Lop Nor to AAK paths. Our third method, combined source and path corrections, has only been tested at WMQ (primarily using KTS explosions and northwest China earthquakes), but the method successfully corrects for source-scaling and propagation effects. We favor this combined source and path correction technique over the other correction methods, and we plan to test this method at other Asian stations.
\end{abstract}

Key Words: seismic discrimination, path correction, source correction, China 


\section{OBJECTIVE}

Monitoring the Comprehensive Test Ban Treaty (CTBT) to magnitude levels below 4.0 will require use of regional seismic data recorded at distances of less than $2000 \mathrm{~km}$. One way to improve discriminant performance is to reduce scatter within ratio or phase amplitude measurements by correcting for the effects of source and path. By reducing scatter, separation between earthquake and explosion populations may be increased. Typically, researchers remove ratio-distance trends before forming discrimination plots ( $c f$. Hartse et al. (1997a)). A linear distance correction approximately accounts for attenuation and geometrical spreading, but other path effects, such as partial blockage of $S_{n}$ or $L_{g}$, are not accounted for and contribute to scatter on discrimination plots. Furthermore, trends on spectral and cross-spectral discrimination plots are produced by the effect of source-scaling. Figure 1 shows an uncorrected $P_{g}$ spectral ratio discrimination plot, the distance trend, and the distance-corrected discrimination plot. The remaining trend on the distance-corrected plot is due to source-scaling.

In this paper our objective is to test three different methods of correcting for path effects, and the third method includes a correction for source-scaling. Our first method follows the approach of Zhang et al. (1996). We remove ratio-parameter trends to reduce scatter within earthquake and explosion populations. This approach requires knowledge of the physical parameters along an event-station path, such as topography, basin thickness, and crustal thickness. Our second approach is somewhat more empirical. We examine spatial distributions of phase amplitudes after subtracting event magnitude and correcting for path distance. For a given station, phase, and frequency band, we grid and then smooth the magnitude-corrected and distance-corrected amplitudes to create a map representing a correction surface. Our third approach to path corrections is the most complicated, but also the most rigorous. For a given station and phase, we invert the spectra of a number of well-recorded earthquakes for source and path parameters. We then use the values obtained from the inversion to correct phase amplitudes for the effects of source size, distance, and attenuation.

We find that simple path parameter corrections can improve discrimination performance along some paths (such as Kazkh Test Site (KTS) to WMQ), but for other paths (such as Lop Nor to AAK) the path parameter corrections are not beneficial. We find that the empirical path correction surfaces improve discrimination performance for Lop Nor to AAK paths. Our combined source and path correction method has only been tested at WMQ (primarily using KTS explosions and northwest China earthquakes), but the method successfully corrects for source-scaling and propagation effects. We favor this combined source and path correction technique over the other correction methods.

\section{RESEARCH ACCOMPLISHED}

\section{Data Used and Region Studied.}

Most of the waveforms used in this study were recorded at WMQ in northwest China and AAK in Kyrgyzstan. However, we have also used data recorded at CDSN stations $\mathrm{LZH}, \mathrm{ENH}$, and XAN, and NIL in Pakistan recorded at distances of up to $2500 \mathrm{~km}$ (Figure 2). Most events studied are regional earthquakes, but WMQ has recorded over 25 KTS explosions (at a distance of about $960 \mathrm{~km}$ ), and AAK has recorded 7 Lop Nor nuclear explosions (at a distance of about $1190 \mathrm{~km}$ ). We obtained event locations, magnitudes, and origin times from United States Geological Survey Preliminary Determination of Epicenter (PDE) catalogs for the years 1987-1996, and we obtained event information from regional Chinese catalogs for earthquakes smaller than about magnitude 4.0 for the years 1987-1989 (Gao and Richards, 1994). 


\section{Method 1 - Correcting Ratios Using Physical Path Parameters.}

Zhang and Lay (1994) showed that statistical trends exist between topography and some phase amplitude ratios for nuclear explosions from the Former Soviet Union's Kazakh Test Site (KTS) recorded at several seismic stations distributed throughout Eurasia. Later, Zhang et al. (1996) obtained a reduction in variance of earthquake phase ratios from the western United States by correcting for surface roughness. While these earlier studies have shown that variance reduction within an earthquake-ratio population is possible, they do not address whether separation between earthquakes and explosions can actually be increased by correcting for path effect. Here, we examine the scatter within each event population and we measure the change in separation between earthquake and explosion populations after correcting $P_{g} / L_{g}$ ratios for path effects related to topography, basin thickness, and crustal thickness (Figure 3 ).

For northwest China earthquakes and nuclear explosions from KTS recorded at WMQ, we find that distance-weighted topography and distance-weighted basin thickness corrections do indeed improve the $P_{g} / L_{g}$ discriminant for the 1.5-3 and 2-4 $\mathrm{Hz}$ bands. We speculate that this increased separation occurs because the explosions from KTS traverse paths with relatively smooth topography and uniform crustal thickness, while most earthquakes we studied have more complicated paths, traversing the topographically rough Tian Shan, the Tarim Basin, and the Junggar Basin (Figure 2). For northwest China earthquakes and nuclear explosions from the Lop Nor test site recorded at AAK, we do not find path corrections that improve the $P_{g} / L_{g}$ discriminant. For details concerning this study see Hartse et al. ( 1997b). The primary drawbacks of this method is the required reliance on physical path measurements, which may often be quite coarse, and the fact that we have not accounted for source-scaling effects.

\section{Method 2 - Empirical Path Corrections.}

We have examined spatial distributions of amplitudes of regional phases to find coherent patterns that can be used as path corrections (Phillips $e$ t al., this volume). For data recorded at a given station, we first correct phase amplitude for source size by subtracting event magnitude $\left(m_{b}\right)$, followed by a linear distance correction. The corrected amplitudes are then smoothed spatially by averaging values over a geographical grid (Figure 4). We smooth only where a threshold number of crustal events (currently 5) fall inside a given bin. Our implicit assumption is that the smoothing averages out source radiation and depth effects, leaving an estimate of the path effect that can be used for correcting phase amplitudes and, therefore, individual discriminants.

After estimating correction surfaces for several phases and frequency bands, we use the correction maps to form amplitude ratios and then discrimination plots. Figure 5 shows results for Lop Nor events recorded at AAK. For the $P_{g} / L_{g}(3-6 \mathrm{~Hz})$ band, the corrections increase separation between the earthquakes and explosions. There are two drawbacks to this method. First, correction maps can only be estimated when there are abundant earthquakes from a given region. Second, we have still not considered the source-scaling effect that will be present in the spectral and cross-spectral ratios.

\section{Method 3 - Source and Path Amplitude Corrections.}

Our third correction technique is a procedure for the estimation of frequencydependent source and propagation amplitude corrections for regional seismic discriminants (Source Path Amplitude Correction - SPAC; Taylor and Hartse, 1997). For a given station and phase, a number of well-recorded earthquake spectra are inverted for source and path corrections. The method assumes a simple Brune (1970) earthquake-source model and a simple propagation model consisting of a frequency-independent geometrical spreading and 
frequency-dependent power-law $Q$. The inverted low-frequency levels are then regressed against $m_{b}$ to derive a set of corrections that are a function of $m_{b}$ and distance. Once a set of corrections are derived, effects of source scaling and distance as a function of frequency are applied to amplitudes from new events prior to forming discrimination ratios. The resulting discriminants are normally distributed and amenable to multivariate feature selection, classification, and outlier techniques.

To date, most discrimination studies have removed distance corrections once a particular amplitude ratio is formed (Distance Corrected Ratio - DCR; Figure 1). DCR generally works well for phase ratios taken in a particular frequency band. However, when different frequency bands are combined (for phase spectral ratios or cross spectral ratios), significant source-scaling effects (e.g. corner-frequency scaling) can remain, causing the discriminants to vary as a function of event size and to be non-normally distributed. It is then often necessary to construct non-physical transformations in an attempt to make the discriminants multivariate normal. The SPAC technique can be used to construct discriminants that are multivariate normal. Moreover, phase amplitude residuals as a function of frequency can be spatially averaged and used as additional path-specific corrections to correct for additional propagation effects, such as phase blockages.

For the frequency-dependent amplitude inversion, we basically follow the technique of Sereno et al., (1988) with minor modifications. The results of the inversion for the source-path corrections for $L_{g}$ are shown in Figure 6 . Once the parameters are obtained, the amplitudes from other events can be corrected for source scaling and propagation using SPAC. To do this, it is first necessary to obtain an estimate of the low-frequency level, $S_{0}$. We have chosen to tie the low-frequency level to magnitude. Note that although the dislocation source model used in correcting the amplitudes is not appropriate for nuclear explosions, we still use it because, in an actual monitoring situation, the source type would be unknown.

As an example application, we corrected $P_{g}$ amplitudes and plotted $P_{g}(0.75-1.5 / 4-$ 8) $\mathrm{Hz}$ spectral ratios versus $m_{b}$. The top portion of Figure 7 shows the distance-corrected spectral ratio (this correction is illustrated in Figure 1) and the SPAC-corrected ratio. For the upper left figure, we formed $P_{g}$ spectral ratios for earthquakes with good signal-tonoise ratio, and then regressed the ratios against the logarithm of distance. We then applied the distance correction factors to all ratios for earthquakes and nuclear explosions having signal-to-noise values greater than 2 . The corner frequency scaling is still present as an increase in the spectral ratio with magnitude. The overlap between the two populations is illustrated in the lower left portion of the figure using the estimated normal probability density function. Visually, the $P_{g}$ ratio with just the distance correction plotted versus mb appears to show good separation between earthquakes and explosions. However, $m_{b}$ is never actually used as a discrimination variable and a multivariate discrimination method would see the projection of the spectral ratio on the ordinate. For the SPAC method, the trend with $m_{b}$ is removed and the separation and variance projected on the ordinate are improved and reduced, respectively (compare Figure 7 lower left with lower right).

\section{CONCLUSIONS AND RECOMMENDATIONS}

We tested three path correction methods for regional seismic discriminants. The first method removes the effects of particular physical parameters along a given path, such as topography or crustal thickness. The advantage of this method is that it is easy to apply, and it can be applied where little historical seismicity has been recorded. However, in many regions, some physical parameters are poorly known, such as basin thicknesses. Also, for northwest China we did not find consistent corrections that work for stations 
AAK and WMQ, two relatively "close" stations for this region of Asia. Our second method is an empirical path correction, relying solely on the residuals of phase amplitudes. Based on initial results, this method has helped increase $P_{g} / L_{g}$ discriminant performance. However, this method relies on abundant data and hence will not be applicable to relatively aseismic regions. Our final method corrects for both source-scaling effect and propagation effects. We have used this method to improve the $P_{g}$ spectral ratio discriminant using data from WMQ. We favor this correction technique as it removes source-scaling effect and propagation effect. We plan to apply this technique across central Asia.

\section{REFERENCES}

Brune, J. N. (1970). Tectonic stress and spectra of seismic shear waves from earthquakes, J. Geophys. Res., 75, 4997-5009.

Fielding, E. J., M. Barazangi, and B. L. Isacks (1993). A geological and geophysical data base for Eurasia, Final Technical Report, ARPA NMRO F29601-91-K-DB08.

Gao, L. and P. G. Richards (1994). Studies of earthquakes on and near the Lop Nor, China, nuclear test site, Proceedings of the 18th Annual Seismic Research Symposium on Monitoring a Comprehensive Test Ban Treaty, 106-112.

Hartse, H. E., S. R. Taylor, W. S. Phillips, and G. E. Randall (1997a). Preliminary study of seismic discrimination in central Asia with emphasis on western China, Bull. Seis. Soc. Am., 87, 1464-1474.

Hartse, H. E., R. A. Flores, and P. A. Johnson (1997b). Correcting regional seismic discriminants for path effects in western China, submitted, Bull. Seis. Soc. Am., submitted, June, 1997.

Phillips, W. S., G. E. Randall, H. E. Hartse, S. R. Taylor, and H. J. Patton (1997). Source and path effects on regional phases in China, Proceedings of the 19th Annual Seismic Research Symposium on Monitoring a Comprehensive Test Ban Treaty, (this volume).

Sereno, T. J., S. R. Bratt, and T. C. Bache (1988). Simultaneous inversion of regional wave spectra for attenuation and seismic moment in Scandinavia, J. Geophys. Res., 93, 2019-2035.

Taylor, S. R. and H. E. Hartse (1997). A procedure for estimation of source and propagation amplitude corrections for regional seismic discriminants, submitted, J. Geophys. Res., June, 1997.

Zhang, T. and T. Lay (1994). Analysis of short-period regional phase path effects associated with topography in Eurasia, Bull. Seis. Soc. Am., 84, 119-132.

Zhang, T., T. Lay, S. Schwartz, and W. R. Walter (1996). Variation of regional seismic discriminants with surface topographic roughness in the western United States, Bull. Seis. Soc. Am., 86, 714-725.

Wessel, P. and W. H. F. Smith (1991). Free software helps map and display data, EOS Trans. $A G U, \mathbf{7 2}, 441$.

\section{Acknowledgements}

We thank Howard Patton for a thoughtful review of this paper. We thank the staff at the IRIS Data Management Center for prompt replies to our data requests. Maps were created using the Global Mapping Tool (GMT) (Wessel and Smith, 1991). This work is in support of the DOE Comprehensive Test Ban Treaty Research and Development Program, ST482A, and was performed at Los Alamos National Laboratory under the auspices of the United States Department of Energy, Contract Number W-7405-ENG-36. 


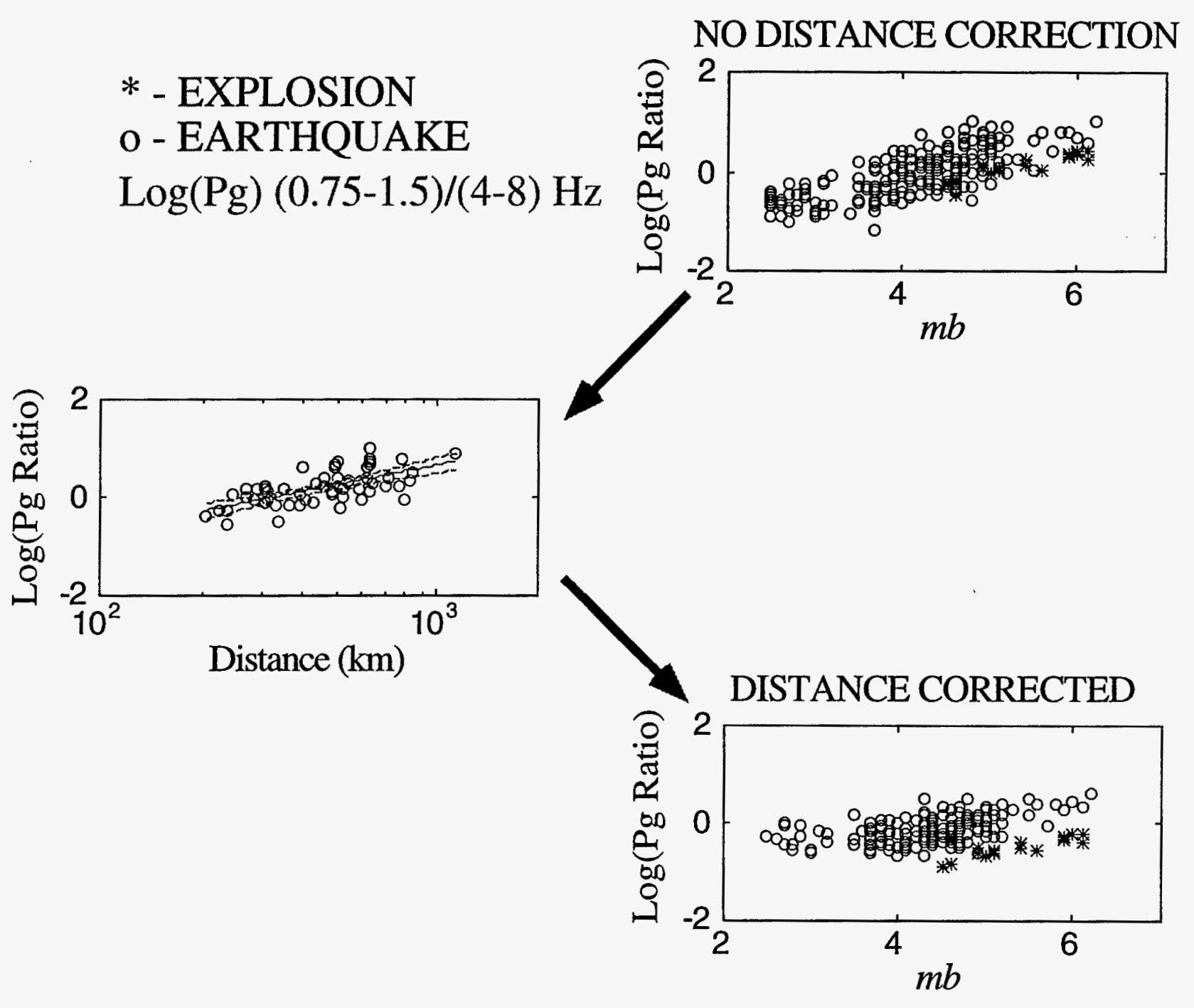

Figure 1. Illustration of the Distance Corrected Ratio (DCR) technique commonly used for applying distance corrections to a Pg spectral ratio discriminant. Upper right panel shows uncorrected spectral ratios for earthquakes and nuclear explosions at WMQ (Hartse et al., 1997a). Left panel shows earthquake $P_{g}$ spectral ratios for events with a signal-tonoise ratio greater than 10 regressed versus the logarithm of the distance. Lower right panel shows distance-corrected $P_{g}$ spectral ratio. Notice dependence of the spectral ratio with magnitude remains. 

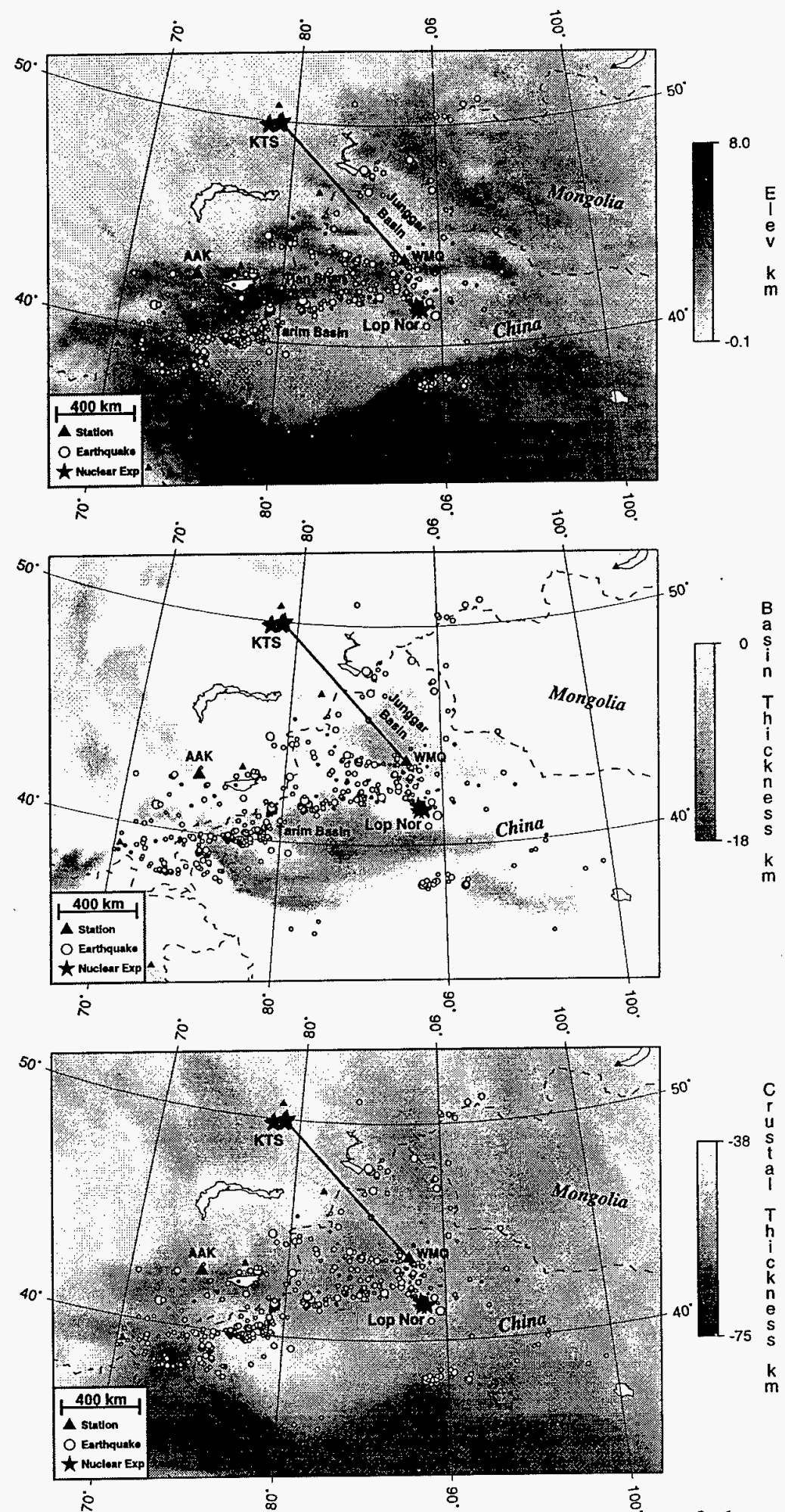

Figure 2. Topography, basin thickness and crustal thickness maps of the northwest China region. Nuclear explosions and earthquakes used in this study were recorded at WMQ and AAK. The heavy line marks the path from the Kazakh Test Site to WMQ. Gridded data obtained from the Cornell Database (Fielding et al., 1993). Parameters displayed and extracted from data grids using the GMT software of Wessel and Smith (1991). 

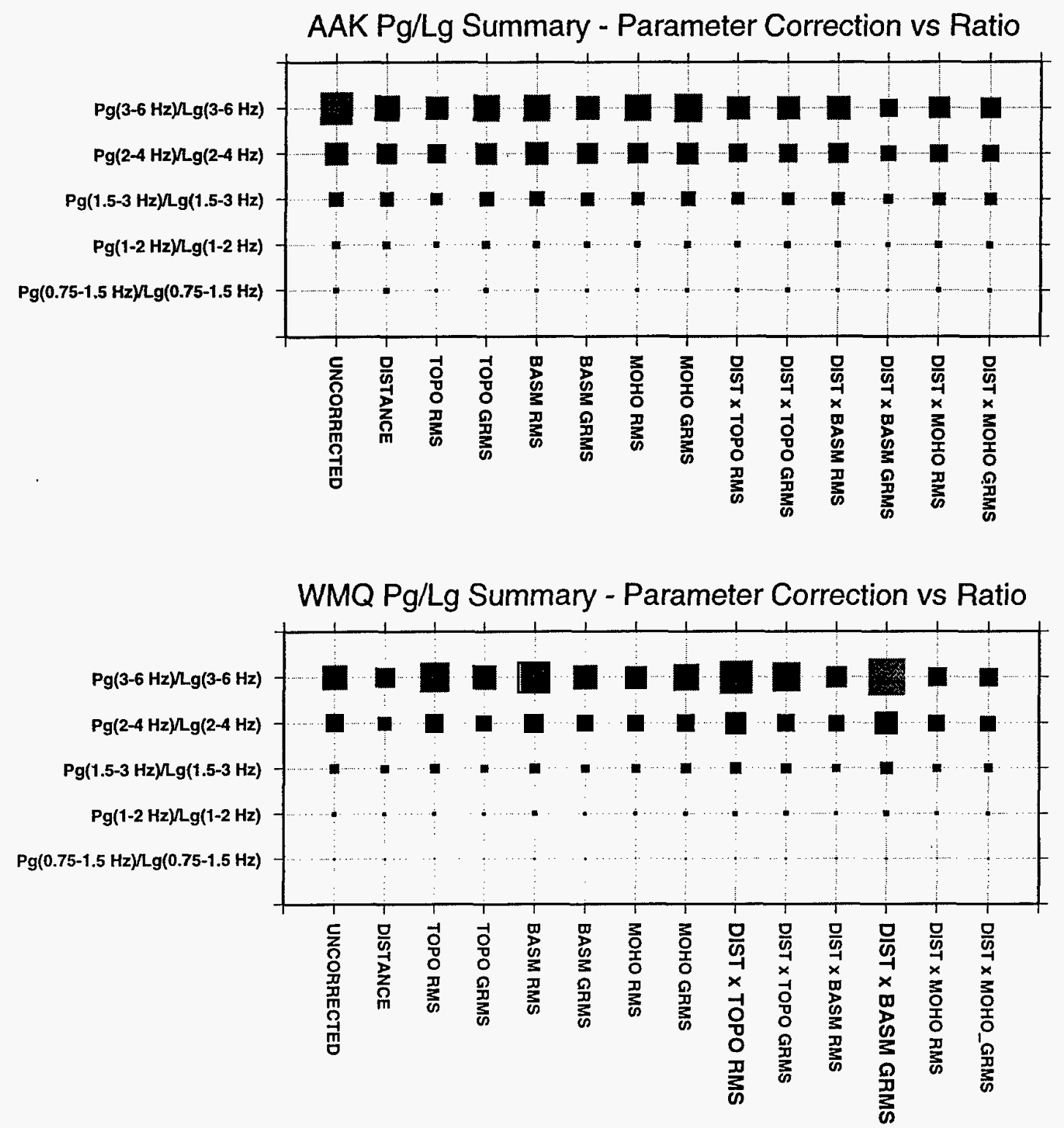

Figure 3. Summary discrimination plots for $P_{g} / L_{g}$ ratios at AAK and WMQ for various physical parameter corrections. Bands tested are indicated along the vertical axis. The size of each square is scaled to the Mahalonobis Distance $\left(\Delta^{2}\right)$, a function of separation between population means $(\bar{v})$ and variance within each population $\left(\sigma^{2}\right)$ : $\Delta^{2}=\left(\bar{v}_{x}-\bar{v}_{q}\right)^{2} /\left(\sigma_{x}^{2}+\sigma_{q}^{2}\right)$. Actual values range from 0.03 to 14.6. As has been shown previously, as $f$ increases, the $P_{g} / L_{g}$ discriminant performance also increases. The most successful corrections for WMQ are distance-weighted roughness of the basin thickness gradient (DIST x BASM GRMS) and the distance-weighted roughness of the topography (DIST $\mathrm{x}$ TOPO RMS). For AAK we found no corrections which increase Mahalonobis Distance relative to the uncorrected case. 


\section{Smoothed, Distance-Corrected Log A - mb:}

LZH BHZ Lg $0.75 \mathrm{~Hz}$

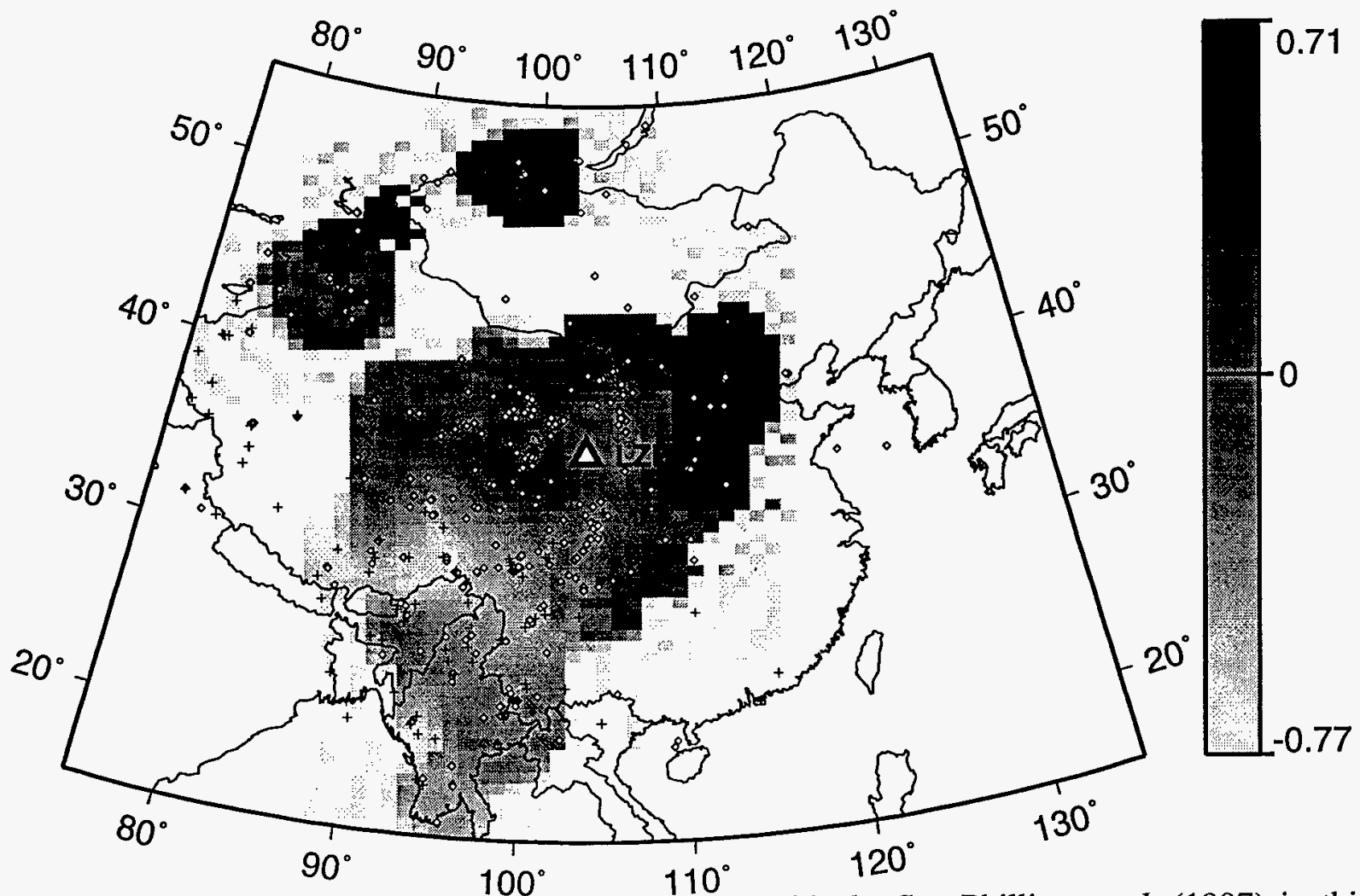

Figure 4. Path correction map of $L_{g}$ amplitude residuals. See Phillips et al. (1997) in this proceedings volume for more details.

Discriminant Ratios:

\section{AAK BHZ, Pg 3.0-6.0 Hz/Lg 3.0-6.0 Hz}
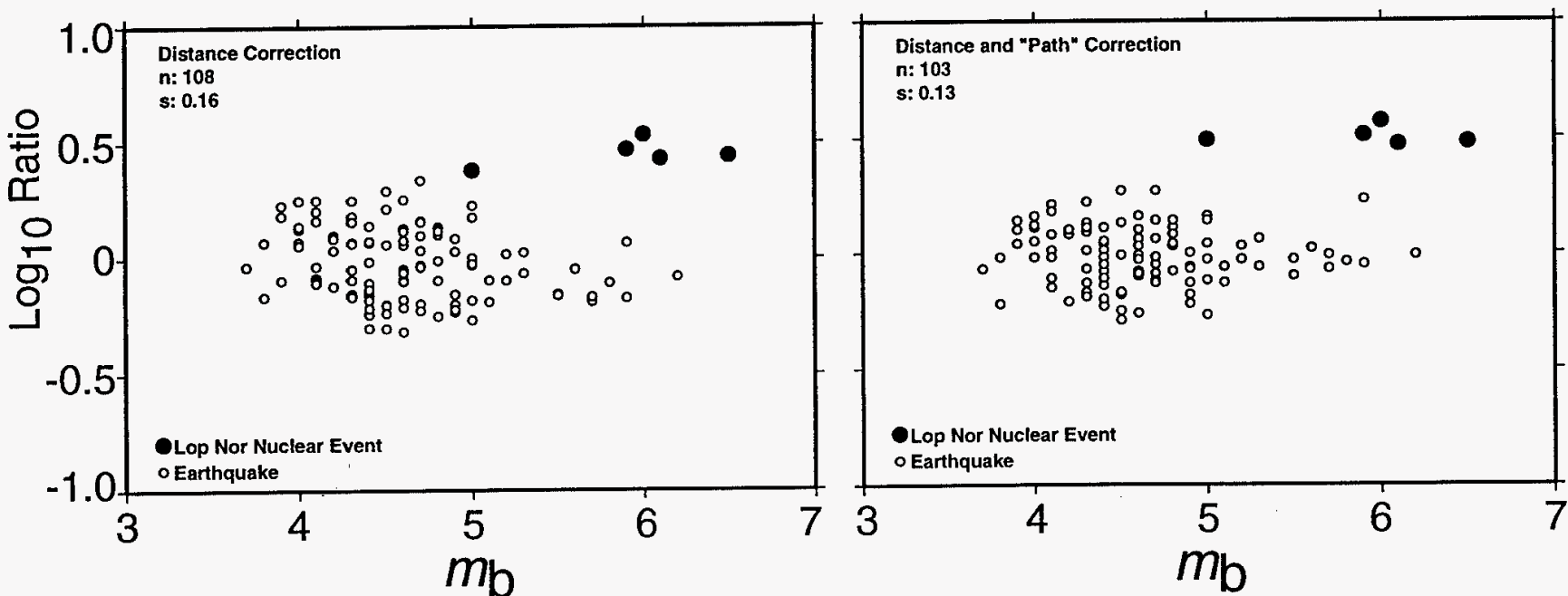

Figure 5. Discrimination plots for the $P_{g} / L_{g}(3-6 \mathrm{~Hz})$ ratio before (left) and after (right) empirical path corrections have been applied. The path corrections have increased separation between northwest China earthquakes and Lop Nor explosions. 

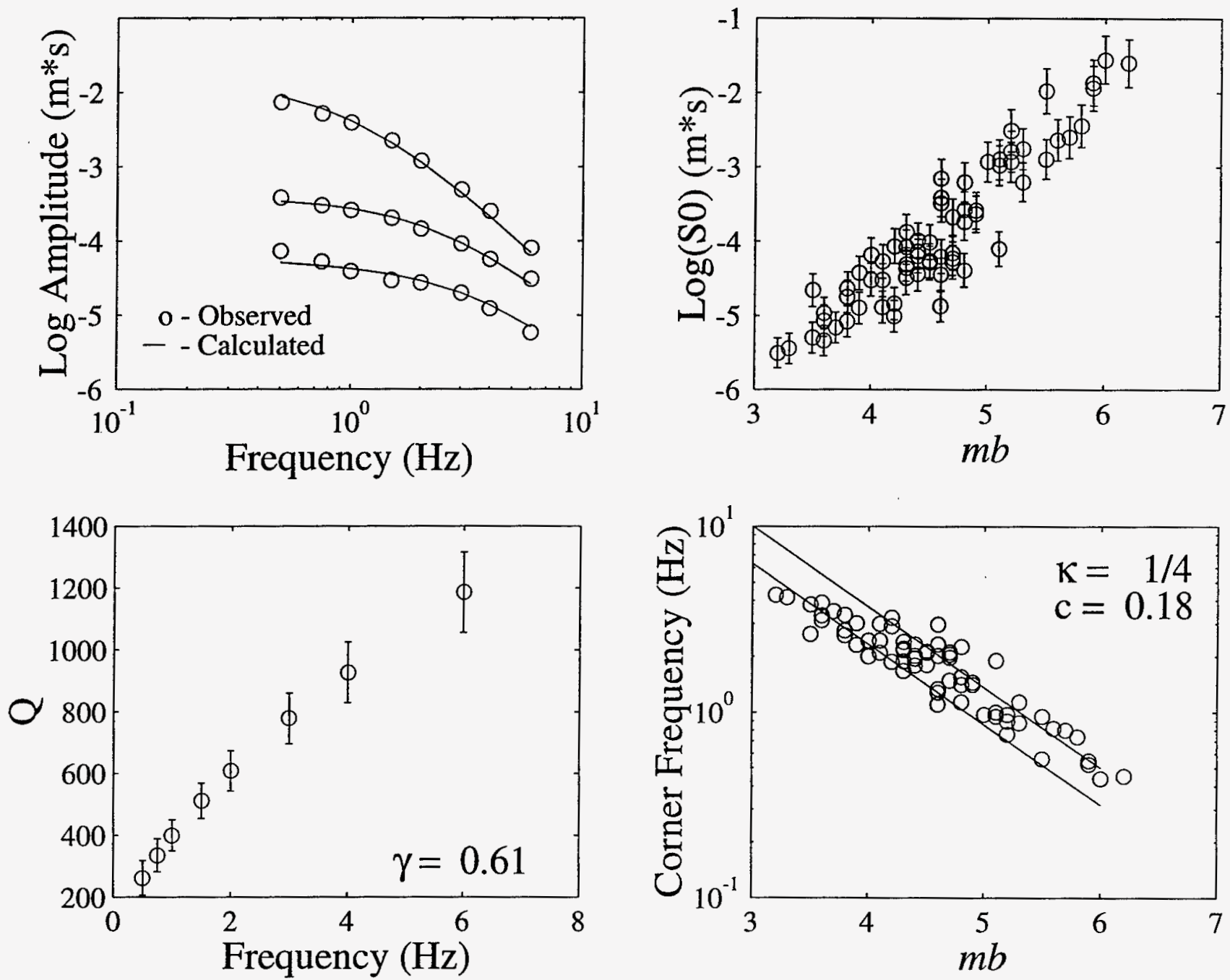

Figure 6. Results from inversion of earthquake $L_{g}$ pseudo spectrum. Geometrical spreading factor $(\eta)$ assumed to be $1 / 2$. Upper left; calculated and observed spectra for 3 randomly chosen events. Lower left; frequency-dependent $Q$ values, $95 \%$ confidence limits, and $\gamma\left(Q_{0}\right.$ set to 400$)$. Upper right; logarithm of the low-frequency level, $S_{0}$, versus $m_{b}$ and $95 \%$ confidence limits. Lower right, corner frequency versus $m_{b}$. Also shown are theoretical curves for S-wave corner frequency using a Brune (1970) dislocation source model (where $\kappa=1 / 3$ ) for stress drops of 5 and $20 \mathrm{MPa}$ (50 and 200 bars; lower and upper curves, respectively). 


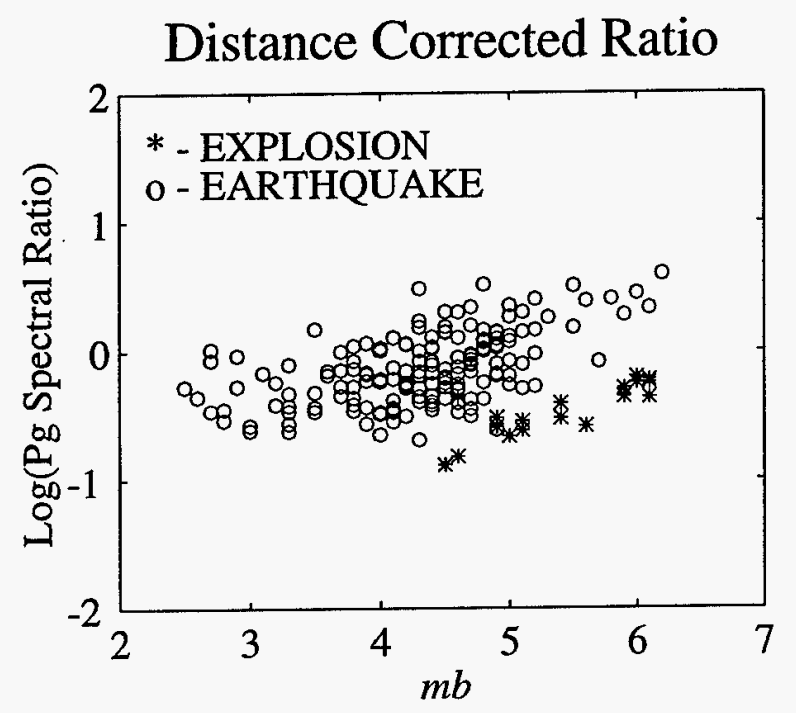

Source and Path Amplitude Correction
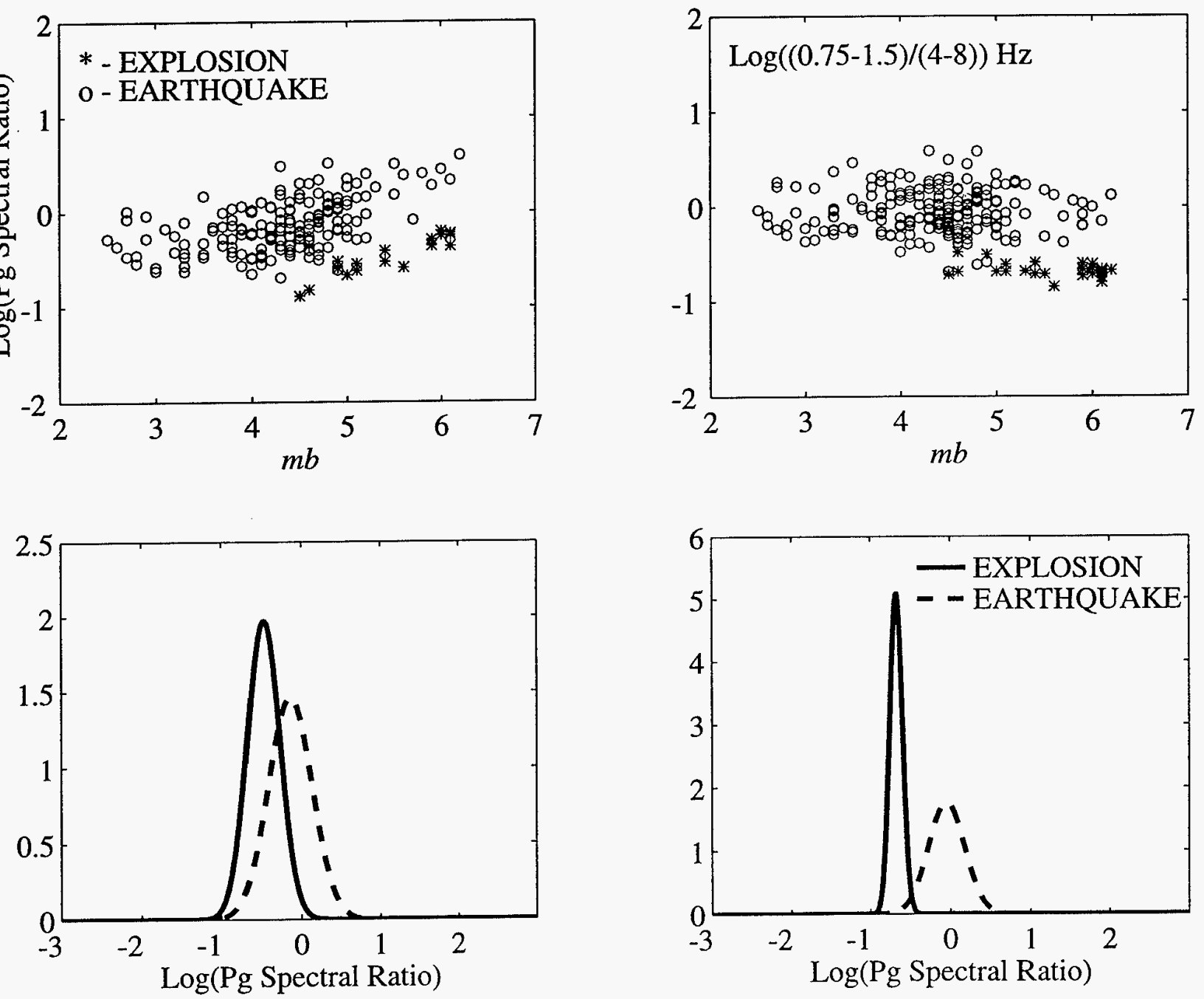

Figure 7. Comparison of DCR (left) and SPAC (right) techniques. $P_{g}$ spectral ratio of the 0.75 to 1.5 and 4 to $8 \mathrm{~Hz}$ bands versus $m_{b}$ using the distance correction method illustrated in Figure 1 (top left) and using the combined source and path corrections (upper right) for earthquakes and nuclear explosions described in Hartse et al., (1997a). Normal probability density function for $P_{g}$ spectral ratio of earthquakes and nuclear explosions using just distance correction (lower left) and combined source and path corrections (lower right). 
Mgroorit

DDE 19971202073 